\title{
La Educación para la Ciudadanía Global: Una apuesta por la Democracia
}

\author{
Education for Global Citizenship: Promoting Democracy
}

\author{
Manuela Mesa * \\ CEIPAZ e Instituto DEMOSPAZ-UAM, España
}

\begin{abstract}
En este artículo se hace una reflexión sobre el papel de la educación en el actual contexto internacional, marcado por profundos cambios y por una situación de incertidumbre e inestabilidad y de retroceso en derechos. Se abordan los diversos marcos interpretativos que explican el mundo actual y que se articulan en torno al eje global-local y al eje identitario. En un mundo caracterizado por la complejidad y la rapidez de cambios es muy importante superar la compartimentalización del conocimiento en áreas estanco (matemáticas, sociales, lengua, etc.) para abordarlo de manera global. Desde el punto de vista cognitivo, se plantea la necesidad de superar la mera información aislada o la simple enumeración de datos, estableciendo conexiones causales entre los diversos acontecimientos y realidades y elaborando un discurso explicativo de la realidad que interrelacione la paz, los conflictos, los derechos humanos y la sostenibilidad ambiental, entre otros. Desde el punto de vista metodológico se proponen enfoques para aprehender la realidad, a partir de la utilización de herramientas que combinen aspectos racionales, visuales, emocionales y numéricos y que estimulen la creatividad y la "imaginación moral". Y desde las estrategias de acción, la práctica educativa debe superar el marco escolar y tener en cuenta otros ámbitos educativos no formales y en estrecha colaboración con las organizaciones sociales, abordar los grandes problemas globales-locales. La educación como práctica transformadora no puede estar al margen de los grandes retos que enfrenta la humanidad en el ámbito nacional y multilateral. La Agenda 2030 y los Objetivos de Desarrollo Sostenible abre oportunidades para consolidar la educación para la ciudadanía global.
\end{abstract}

Descriptores: Globalización; Educación para la ciudadanía global; Agenda 2030; Transformación social.

This paper makes a reflection on the role of education in the current international context, marked by profound changes and by a situation of uncertainty and instability and reverse in rights. It discusses the various interpretative frameworks that explain the current world and that are articulated around the global-local axis and ethnic-national identities and multiple identities. In a world characterized by the complexity and speed of changes, it is very important to overcome the the compartmentalization of knowledge in areas such as mathematics, social, language, etc and look for global approaches. From the cognitive point of view, there is a need to modify the way of organizing the knowledge and put it in relation to new concepts. It's about creating emancipatory narratives that they put in the centre of the human person and the relationship between peace, development, human rights and justice. From a methodological point of view, it proposes strategies to apprehend reality, from the use of tools that combine rational aspects, visual, emotional and numeric and that stimulate creativity and the "moral imagination". And from the strategies of action, educational practice must overcome the school framework and taking into account other areas to non-formal education and in close collaboration with social

*Contacto: mmesa@ceipaz.org

ISSN: 2254-3139

www.rinace.net/riejs/ revistas.uam.es/riejs
Recibido: $\quad 2$ de enero 2019

$1^{\text {a }}$ Evaluación: 28 de enero 2019

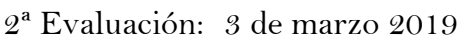

Aceptado: $\quad 4$ de abril 2019 
organizations, to deal with the large global-local problems related to violence, inequality, environmental sustainability and global warming. Education as a transformative practice cannot be outside of the major challenges facing humanity at the national and multilateral. The 2030 Agenda and the Sustainable Development Goals gives opportunities for the consolidation of the Education for Global Citizenship.

Keywords: Globalization; Education for global citizenship; 2030 agenda; Social transformation.

\section{Introducción}

Una de las preocupaciones de la última década ha sido definir el papel que juega la educación en el actual contexto internacional caracterizado por la intensificación de los procesos de globalización. Se trata de definir cuáles deberían ser los fines, objetivos principales y que tipo de conocimientos y habilidades son necesarios para hacer frente a los problemas locales que se manifiestan en el plano local y a los desafíos locales que afectan en el ámbito global. En definitiva, como promover la formación integral de la persona en un mundo cada vez más globalizado. La educación para la ciudadanía global remite necesariamente a la globalización y a los cambios que se han producido en la actual situación.

El periodo actual es incierto e impredecible y ya no sirven los marcos interpretativos del periodo anterior que nos ayudaban a entender los acontecimientos. En los últimos años se ha experimentado un cambio profundo en el contexto internacional, con extraordinarios retos globales que afectan a todos los ámbitos de la sociedad, desde la política, la economía, el desarrollo, la cultura y la educación, entre otras cuestiones. Se observan transformaciones en las estructuras económicas y sociales y en las pautas de distribución del poder en las que se sustenta el sistema internacional; estos cambios se iniciaron con la crisis económica global en 2008 y han tenido como consecuencia el freno o fracaso de los procesos de globalización económica y un retroceso en la democracia liberal (Sanahuja, 2018 p. 42)

El fracaso de la globalización y su lado oscuro, muestra el poder desmesurado de los poderes financieros, las inmensas asimetrías que se producen en las sociedades, en las que aumentan la desigualdad en un proceso de concentración de la riqueza que no tiene precedentes. Esta situación supone un enorme desafío para las democracias liberales, al no ser capaces de dar respuesta a los problemas que enfrentan la ciudadanía y que han generado un gran malestar y desafección hacia un sistema político que cada vez resulta más ineficaz y alejado de las necesidades de la población. El resultado ha sido el ascenso de gobiernos autoritarios y de ultraderecha que han sabido movilizar y canalizar el malestar social, el miedo y la incertidumbre para ganar peso electoral, poder parlamentario e incluso formar gobierno en algunos países. Desde Donald Trump en Estados Unidos, a Jair Bolsonaro en Brasil, pasando por los gobiernos dictatoriales en el Oriente Medio, después de las fallidas "primaveras árabes", y el ascenso de la ultraderecha en Europa, se consolida una tendencia general que se expresa en el recorte de derechos y libertades y en la deslegitimación de las instituciones democráticas. Todo ello tiene graves consecuencias para la democracia y afecta también a los sistemas educativos.

Esto se produce en un momento de grandes retos globales, relacionados con el calentamiento global, con el aumento de la cifra de personas refugiadas y migrantes, o con el cambio en el modelo productivo, que está destruyendo miles de empleos, entre otros 
fenómenos de extrema gravedad. Esta situación requiere de una política concertada en el ámbito multilateral y de medidas urgentes en el plano nacional y regional y se pone de manifiesto los límites de la globalización como modelo universalizable.

En este escenario de cambio e incertidumbre se plantea si la globalización ha logrado un mayor bienestar y seguridad de la población mundial, o bien ha supuesto el aumento de la brecha entre los más ricos y pobres, que se ha acrecentado de manera global y de forma constante. Nos enfrentamos al dilema de optar por respuestas que abrazan el nacionalismo excluyente y proponen respuestas centradas en lo local; o bien respuestas cosmopolitas basadas en la expansión de derechos en el marco multilateral y como parte de la Agenda 2030 de desarrollo sostenible y los acuerdos sobre cambio climático. Algunos autores/as proponen diversos marcos interpretativos para analizar el contexto actual a partir de nuevos ejes o clivajes, en torno a posiciones favorables o contrarias a la globalización y entre posiciones de izquierda y derecha (Sanahuja 2018; Vallespin y Bascuñan, 2017).

Estos marcos interpretativos son importantes porque nos ayudan a visualizar distintas maneras de explicar la realidad y parten de concepciones distintas sobre el conocimiento y sobre el papel que juega la educación en la sociedad. Como han desarrollado ampliamente los socio-constructivistas y desde la pedagogía crítica, los discursos narrativos explican la realidad, ordenan la historia y ubican los acontecimientos colectivos dentro de una unidad coherente que incluye el pasado y establece una "memoria" que comparten todos los individuos socializados en una colectividad (Berger y Luckmann 1968, p. 33). Proporcionan un sentido de propósito a las prácticas sociales, definen expectativas, asignan roles y funciones y prescriben comportamientos y normas. También tienen un importante papel constitutivo de los intereses, valores e identidades de los actores sociales y políticos.

Este análisis resulta de gran interés para el ámbito educativo, porque permite analizar las distintas visiones o formas de explicar el mundo en el que vivimos y explicitar donde se sitúa la educación para la ciudadanía global. También permite abordar las tensiones entre lo global y local, o entre identidades étnico-nacionales e identidades múltiples en un marco más amplio que lleva consigo acciones e iniciativas muy diversas.

\section{La tensión entre lo local y global: El sentido de pertenencia a una comunidad de ciudadanos/as en lo local y en lo global}

Las posiciones frente a la globalización han conformado diversas narrativas, entre los que la promueven y la consideran un factor de progreso y aquellos que se oponen centrándose en sus consecuencias. Entre los favorables a la globalización, por una parte, se encuentran aquellas visiones que defienden el statu quo y el neoliberalismo y son favorables a la democracia liberal, al libre mercado y a la empresa privada; aquellos que consideran que el mercado se autorregula, por lo que no es preciso establecer leyes, ni al comercio, ni a las transacciones financieras. Su más clara expresión es el Foro Económico de Davos y promueven grupos plutocráticos como el G-7 o el G-20 que funcionan al margen de Naciones Unidas.

Sin embargo, el fracaso de la globalización neoliberal, el aumento creciente de la desigualdad y la exclusión y el empobrecimiento de amplias capas de las clases medias en 
los países desarrollados ha llevado a un auge del nacionalismo excluyente, en la defensa del interés nacional, desentendiéndose de los problemas globales y considerando una amenaza todo aquello que proceda del exterior. Son los "perdedores de la globalización", en los que el miedo y la inseguridad, se ha convertido en el caldo de cultivo para la emergencia de los movimientos de ultraderecha.

Para los globalistas de Davos, la educación se concibe como un instrumento al servicio del mercado, que debe formar a las potenciales personas consumidoras y ajustarse al mercado laboral, desde un planteamiento individualista y competitivo orientado por el éxito y el rendimiento económico. Asumen la racionalidad positivista, considerando el conocimiento como objetivo, neutral, cuantificable y estandarizado. Se cuestiona la educación pública al considerarla ineficaz y de baja calidad.

Por otra parte, están aquellos cuya visión del mundo es neokantiana y se basan en el cosmopolitismo; plantean la necesidad de regular la globalización a partir de una legislación que permita la protección de los derechos humanos, laborales, sociales y del medio ambiente. Proponen la preservación de los bienes públicos globales relacionados con la educación, la salud, la cultura y el medio ambiente y defienden el multilateralismo democrático para abordar la lucha contra la pobreza y la desigualdad. Y defienden la Agenda 2030 como resultado de un gran consenso internacional para abordar estos problemas globales. Este es en este marco donde se situaría la educación para la ciudadanía global.

La globalización y la democracia cosmopolita hacen un cuestionamiento de aquellas realidades sociales y culturales que segregan a los individuos y los grupos humanos por su categoría de clase, estatus o género, que a su vez se traducen en relaciones sociales de dominación y subordinación. Esta propuesta tiene una importante dimensión normativa, dado que se trata de ideales, o de un imperativo ético, moral y político, que permite la reconstrucción de la teoría y la práctica democrática en la era de la globalización.

Es así, como la noción de ciudadanía, ligada a un sentimiento de pertenencia a una comunidad global va ligado a unos derechos y deberes que adquieren una dimensión supraestatal. La consecución de una ciudadanía cosmopolita exige en los que se refiere a los derechos políticos, democratizar el "espacio global" y en los que se refiere a los derechos sociales, dotar a la justicia de una dimensión planetaria. Y por ello la solidaridad, la lucha contra la pobreza y la desigualdad se sitúan en el centro de las políticas y es una responsabilidad que forma parte de los compromisos internacionales adquiridos por cada gobierno.

El estatus de ciudadanía se constituye como un elemento unificador e integrador de la sociedad. No es sólo un estatus que reconoce unos derechos políticos sino también un proceso y una práctica por la que la ciudadanía comparte unos valores y normas de comportamiento que posibilitan la convivencia y les dota de una identidad colectiva, en este caso una identidad colectiva local-global. Por otro lado, el ejercicio de la ciudadanía global se basa en la idea de universalidad, en la que la democracia defiende los mismos derechos para todos los individuos. 


\section{La tensión entre identidades etno-nacionales y las identidades múltiples}

El fracaso de la globalización ha tenido como consecuencia la emergencia con fuerza un nacionalismo excluyente, de carácter racista y xenófobo que niega derechos a los que no forman parte de la nación y que trata de romper los lazos con la globalización. America First en expresión del presidente de los Estados Unidos, Donald Trump replicado después por otros gobiernos, cuestiona esta idea de cosmopolitismo que está en la base de la noción de ciudadanía global. Asimismo, los llamados valores "patrios" son también una manera de trazar fronteras entre el "nosotros" y "ellos", asociando a naciones determinadas valores universales que forman parte de un acervo político y social que ha logrado de la historia ha alcanzado la humanidad.

Se trata de un "racismo de franquicia", que forma parte de una tendencia internacional y que de manera muy inteligente se adapta a las peculiaridades de cada lugar (Fernández de Castro y González-Páramo, 2019). Se impone en el discurso, conceptos excluyentes que naturalizan la discriminación y promueven la violencia (Stanley, 2019). Se analiza la realidad desde un enfoque de darwinismo social, en clave de ganadores y perdedores, y situando los valores de solidaridad y justica social, como un signo de debilidad propio de los "perdedores". Asimismo, se utiliza el mito de la superioridad nacional para movilizar y actuar asociado a un concepto de identidad nacional, que resalta los esencialismos de manera acrítica.

Se deja en un segundo plano otros problemas globales relacionados con el cambio climático, la desigualdad o la violencia. Entre los grandes problemas actuales, dan una especial relevancia a la inmigración y se criminaliza al inmigrante y se le responsabiliza de la inseguridad y de poner en riesgo la identidad. En definitiva, son discursos que promueven la polarización y la provocación y que se apoyan en las emociones colectivas de inseguridad y rechazo para movilizar a las personas.

Por su parte, el cosmopolitismo acepta la existencia de múltiples identidades que se superponen y, al mismo tiempo, el compromiso de defender la igualdad de todos los seres humanos. Las identidades son dinámicas y han sido definidas de múltiples formas, dado que se construyen a partir de los referentes históricos, de las experiencias personales, del contexto en el que se vive y están determinadas por el espacio y el tiempo.

Mientras muchas personas se consideran ciudadanas cosmopolitas, capaces de expresar sus múltiples identidades, otras se sienten atraídas por las identidades etno-nacionalistas y políticas que resultan incompatibles con los principios democráticos. Las personas no tendrían que elegir entre ambas opciones, sino que el ejercicio de la ciudadanía debería incluir esas identidades múltiples, que se complementan entre sí. Se trataría de promover el respeto a la diferencia dentro del respeto de los de derechos humanos. Una ciudadanía global respetuosa con la diversidad, en sociedades abiertas con valores cosmopolitas, en la que coexisten identidades múltiples marcadas por el sexo, la clase, el lugar de nacimiento, etc. Se trata de reconocer la unidad en nuestra diversidad, teniendo en cuenta que somos más iguales que diferentes (De Paz 2007, p. 70).

Estas dos visiones de la globalización corresponden a marcos interpretativos de carácter binario que oponen lo local frente a lo global, o las identidades etno-nacionales frente a las identidades múltiples. Presentan muchas limitaciones y restringen el papel de la 
educación para dar respuesta a los grandes retos globales que enfrenta la humanidad. Plantean, por una parte, como superar los discursos de la polarización y crispación para sustituirlos por el diálogo, la escucha y la argumentación. Y por otra, como abordar la diversidad cultural, como un factor de enriquecimiento y el progreso, en lugar de como un espacio para la confrontación entre mundos culturales distintos. Lo autóctono enfrentado a lo foráneo; se trata de superar los esencialismos identitarios que separan comunidades y marginan al diferente. Todo esto supone un gran reto para una educación comprometida con la transformación social.

La incorporación de la noción de ciudadanía global al ámbito educativo abre oportunidades para buscar soluciones y alternativas a los problemas globales y para reafirmar el papel de la educación como una herramienta de cambio y transformación, orientada a la justicia social y la solidaridad. En un momento de cambio y de gran complejidad, la educación se enfrenta ante el dilema de ser factor de reproducción de los valores dominantes que legitiman el statu quo, o bien en convertirse en un motor de cambio, que busca transformar la realidad injusta y desigual. Como afirma el Informe Delors (Delors, 1997) la educación es un factor indispensable para que la humanidad pueda conseguir los ideales de paz, libertad y justicia social; representa un medio al servicio del desarrollo humano más auténtico y más armonioso y, por tanto, un medio para hacer retroceder la pobreza, la marginación, la opresión y la guerra. La educación tiene, pues, una responsabilidad específica en la construcción de un mundo más solidario. Y por ello, la adopción de la Agenda 2030 aprobada por Naciones Unidas en septiembre de 2015, con 17 objetivos y 169 metas, abre oportunidades y posibilidades de acción a múltiples actores, entre ellos a la comunidad educativa; dado que es el resultado de un consenso internacional en torno al desarrollo económico, social y ambiental y la afirmación de la paz y seguridad como un pilar esencial del desarrollo. El carácter universal de la Agenda 2030 y la necesidad de "localizarla" en relación a las capacidades y retos de cada país y cada localidad o territorio abre extraordinarias oportunidades para dar paso a agendas a la vez locales y globales. La idea de universalidad -entre países y personas- para lograr un futuro más justo, equitativo y sostenible es parte de esta agenda. Y es en este marco, en el que la conformación de una ciudadanía global, construida desde abajo, desde las iniciativas de las organizaciones de la sociedad civil, desde la escuela, juegan un papel fundamental para afrontar estos problemas globales, que forman parte de las experiencias locales del día a día. En la medida que los grupos locales y las redes educativas forman parte de redes internacionales de organizaciones, son más fuertes y tienen mayor impacto sus propuestas.

\section{La dimensión cognitiva de la educación para la ciudadanía global}

Desde el punto de vista cognitivo, la educación para la ciudadanía global deberá ofrecer marcos interpretativos para abordar esta realidad compleja y problemática desde los valores de paz y justicia social. Y así, frente a las narrativas binarias, de "un nosotros" frente a un "ellos" que contraponen el bienestar de la ciudadanía de un país, al bienestar del resto de la humanidad, que reafirman identidades etno-nacionalistas frente a las identidades múltiples, que justifican la discriminación y niegan los derechos de las personas refugiadas y migrantes, el concepto de ciudadanía global es extraordinariamente poderoso y emancipador, por su carácter universalista y pluricultural. Como plantea Morin (1999): "Hay que enseñar a no oponer lo universal a la patria, sino vincular de 
manera concéntrica nuestras patrias familiares, regionales y nacionales e integrarlas en el universo concreto de la patria terrenal" (p. 40).

Esto implica un proceso de redefinición de los contenidos de manera que permitan la comprensión crítica de los fenómenos que están surgiendo como resultado de los procesos de globalización e incorporar categorías analíticas que ayuden a interpretar estas nuevas realidades. Se necesitan nuevos conceptos para nombrar estos fenómenos globales y para interpretarlos a partir de marcos cognitivos que conecten saberes con capacidades y valores de solidaridad, la paz y la justicia social.

Esto requiere modificar la forma de concebir el conocimiento. En un mundo caracterizado por la complejidad, la rapidez de cambios y la imprevisibilidad es muy importante superar la compartimentalización del conocimiento en áreas estanco (matemáticas, sociales, lengua, etc.) para abordarlo de manera global. Se trata de reconsiderar la organización del conocimiento, de ponerlo en relación con nuevos conceptos dentro de marcos interpretativos que conforman narrativas emancipadoras, que ponen en el centro al ser humano y muestran la relación entre paz, desarrollo, derechos humanos y justicia. Y esto hay que hacerlo, incorporando las relaciones entre lo local y global -la dimensión espacialy las relaciones entre pasado, presente y futuro -la dimensión temporal- en el análisis de los procesos globales a nivel político, social, cultural, medioambiental y tecnológico.

También supone de-construir viejos conceptos y resignificarlos, superando esencialismos y determinismos que limitan el análisis y la comprensión. Es muy importante, como plantea Maria Novo (2017, pp. 256-261), superar los enfoques de una ciencia mecanicista, reduccionista y determinista para abrirse a una visión compleja en la que tengan cabida el azar y la incertidumbre. Y pasar a una nueva concepción científica que comprenda la imposibilidad de la separación absoluta entre el observador y el observado. Una ciencia que supere la vieja mirada dual de la modernidad (persona/naturaleza; mente/cuerpo) y deje concebir el mundo como una gran máquina que obedece a leyes deterministas. Considerando el conocimiento como algo que se construye de forma colectiva, incorporando los saberes de múltiples actores y que puede contribuir a la emancipación y a la transformación social. Un conocimiento contextualizado, que es aplicado, que está sujeto a preguntas, que busca la interdisciplinariedad, la visión holística y la búsqueda de sinergias positivas. Y que promueve métodos que permitan aprehender entre las partes y el todo, en un mundo en constante cambio.

Es así, como la educación para la ciudadanía global aborda como cuestiones centrales, las causas y consecuencias relacionadas con la desigualdad en el reparto de la riqueza y el poder en el mundo, así como las desigualdades entre hombres y mujeres como resultado de la discriminación que han sufrido a lo largo de la historia, las violencias, el calentamiento global y la diversidad cultural. Todas ellas cuestiones esenciales en el ejercicio de la ciudadanía global-local o "glocal".

\subsection{Las desigualdades planetarias}

En la Agenda 2030 el ODS 10 plantea reducir las desigualdades en y entre los países. Entre las metas del objetivo se señala: "Garantizar la igualdad de oportunidades y reducir la desigualdad de resultados, incluso eliminando las leyes, políticas y prácticas discriminatorias y promoviendo legislaciones políticas y medidas adecuadas al respecto".

Desde el punto de vista educativo, se trata de alcanzar una mayor comprensión de las desigualdades planetarias existentes en el reparto de la riqueza y del poder, sus causas y 
consecuencias y el papel de las élites y sectores con más poder en la construcción de estructuras más justas. Esto implica abordar problemáticas globales que relacionan el desarrollo con los derechos humanos, el medio ambiente, la paz, etc. Supone un análisis en profundidad de las causas de la desigualdad global y la desigualdad al interior de los propios países, así como de sus consecuencias, y no sólo de sus manifestaciones (pobreza, hambre, catástrofes humanitarias, etc.). Para ello, es necesario promover la adquisición de una estructura conceptual básica sobre el desarrollo, desde un enfoque transversal que incluya conocimientos sobre economía, política, historia, antropología y medio ambiente, entre otros.

\subsection{Las desigualdades de género}

El ODS 5 plantea lograr la igualdad entre los géneros y empoderar a las mujeres y niñas. Entre las metas que se establecen está poner fin a todas las formas de discriminación. Es importante tener en cuenta, que aproximadamente en un tercio de los países en desarrollo las niñas tienen enormes dificultades para matricularse en la escuela primaria y secundaria. Esto se traduce en falta de capacitación y, por tanto, de oportunidades para acceder al mercado de trabajo.

Desde el punto de vista educativo, se trata de abordar la persistencia de la desigualdad entre hombres y mujeres y el impacto de la pobreza en las mujeres y las niñas. De superar la discriminación de las mujeres como algo inherente a la biología. Y abordar cuales son los mecanismos de socialización que siguen actuando de forma diferencial en el ámbito educativo entre hombres y mujeres y que actúan de forma discriminatoria valorando el conocimiento masculino e ignorando la realidad femenina. Se necesita un marco interpretativo que aborde el androcentrismo que impregna los currículos escolares, el lenguaje sexista, el uso de los espacios en los centros educativos y todo aquello que contribuya a visibilizar la discriminación. Se trata de revalorizar "lo femenino" universalizándolo, desarrollando instrumentos analíticos que hagan posible la comprensión de la experiencia específica de las mujeres en todo el entramado socioestructural, y, por otra, combatir sus consecuencias y la propia construcción del género como producto de las relaciones de poder y desigualdad (Maquieira, 2001, p. 167).

\subsection{El conflicto y las violencias}

El ODS 16 plantea promover sociedades justas, pacíficas e inclusivas. Este objetivo ofrece una oportunidad para reflexionar sobre los conceptos de paz, justicia y gobernanza global, así como sus factores constitutivos (Mesa, 2018, p. 31)

Desde el punto de vista educativo, se trata de reconocer el conflicto como algo inherente al ser humano que puede ser destructor o motor de cambio en función de cómo se regule la disparidad de intereses. Promover procesos orientados a la transformación pacífica de los conflictos en el ámbito grupal, local e internacional. También de de-construir formas estructurales de violencia que existen en la sociedad y de superar el paradigma de que el uso de la violencia es inevitable. De promover un conocimiento que recoge el valor de la negociación y el consenso, que es abierto y dinámico, que combina lo discursivo, con lo visual y emocional. 


\subsection{El cambio climático y los retos ecosociales}

En la Agenda 2030 el desarrollo sostenible es un eje esencial y primordial e incorpora diversos objetivos relacionados con el agua, la energía, el calentamiento global, la vida submarina y la vida y los ecosistemas terrestres.

Ante la evidencia de que vivimos en un mundo finito -limitado en recursos, pero también en capacidad de regeneración-, uno de los mayores desafíos es el de redefinir aspectos centrales de la relación del ser humano con el medio en el que vive y del que depende. El capitalismo, con sus resortes de crecimiento económico ilimitado, conduce a discontinuidades y rupturas de enorme calado. Continuar por la senda del extractivismo, el productivismo y el consumismo no es ya una opción, por más que nuestras sociedades apenas se den cuenta de ello (Prats et al., 2017).

Desde el ámbito educativo se debería hacer un análisis de la ecodependencia e interdependencia de la humanidad; y abordar la vinculación entre las consecuencias sociales y las ecológicas de los destructivos procesos socioeconómicos actuales. Asimismo, promover y generalizar nociones como las de justicia ambiental e intergeneracional como herramientas útiles de análisis.

También la toma de conciencia de que ninguna cultura tiene soluciones para todos los problemas vitales que enfrenta la humanidad y de la importancia de una ética intercultural, que promueva el diálogo de saberes entre las distintas culturas, respetando sus diferencias y singularidades y construyendo desde todas ellas una convivencia más justa y feliz. Son esas epistemologías del Sur, en palabras de Boaventura de Sousa Santos,

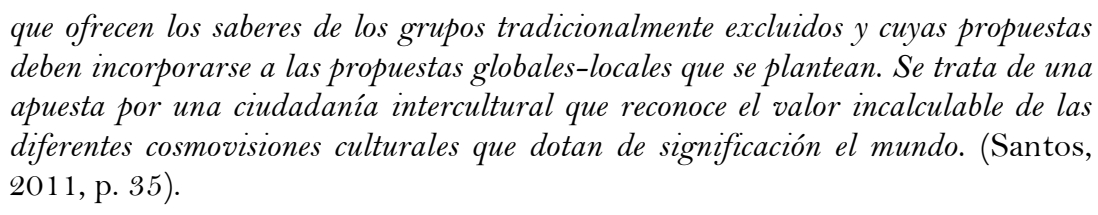

En definitiva, la educación para la ciudadanía global debe promover enfoques integradores, que superen la mera información aislada o la simple enumeración de datos, estableciendo conexiones causales entre los diversos acontecimientos y realidades y elaborando un discurso explicativo de la realidad que interrelacione la paz, los conflictos, los derechos humanos y la sostenibilidad ambiental, entre otros. Sería una forma de "educar para la vida" en palabras de Gimeno Sacristán, que va más allá de la acumulación de conocimientos, sino que ofrece un conocimiento aplicado y que enseña a pensar, a plantearse problemas, a analizarlos, a buscar soluciones alternativas y compararlas y contrastarlas con las opiniones de los otros y a llevarlas a la acción (Gimeno, 2005, p. 15).

\section{Enfoques metodológicos de la educación para la ciudadanía global}

Desde el punto de vista de los procedimientos o metodología se trata de desarrollar un conjunto de acciones centradas en las necesidades del educando, orientadas al desarrollo de habilidades como el pensamiento crítico, la empatía, la capacidad de argumentar, de mediación y negociación y la descodificación de imágenes y mensajes, entre otras. También de la utilización de herramientas que estimulen la creatividad, que permitan analizar la realidad desde distintas perspectivas, combinando los aspectos racionales, 
visuales, emocionales y numéricos, para aprehender esta realidad compleja, llena de matices.

Y, asimismo, potenciar el "pensamiento lateral", entendido como la capacidad para salir de las pautas habituales de percepción, con el fin de buscar una forma de mirar las cosas, de crear nuevos conceptos, nuevos marcos no determinados por pautas rígidas, establecidas o condicionadas con lo que visualizar nuevas posibilidades (Bono, 1999). O bien, promover la "imaginación moral" en palabras de Jean Paul Lederach entendida como: "La capacidad de generar respuestas e iniciativas constructivas que, estando enraizadas en los retos de la violencia, trasciendan y rompan los amarres destructivos". Se trata de desarrollar la capacidad de percibir cosas más allá de la violencia, la necesidad de un acto creativo, capaz de dar a luz algo nuevo, que por su propia existencia provoca cambios en nuestro mundo y en la forma en como lo observamos: "Empiezan a existir cosas nuevas, lo viejo se transforma y aparece nuevas formas de ver". Requiere la capacidad de imaginarnos en una red de relaciones que incluya a nuevos enemigos (Lederach, 2007).

Por último, promover la alfabetización digital; la educación para la ciudadanía global debe capacitar para el uso de internet y de las redes sociales de manera crítica, tanto como herramienta para proveer información, como un medio en sí mismo, que no es neutro y que contiene unos valores y normaliza unas formas de comunicación que deben ser analizados por sus potencialidades para crear redes y una conciencia colectiva y por sus límites cuando difunde discursos de odio y confrontación (Sampedro, 2018). Asimismo, ofrecer claves para identificar las estrategias de desinformación, que cada vez son más frecuentes, con las llamadas fake nerws, que no son noticias y que por el contrario tratan de difundir informaciones y contenidos falsos en redes sociales y plataformas, que amplifican los discursos de odio o contenidos ofensivos (Magallón, 2019).

\section{Estrategias de intervención}

Desde las estrategias de intervención, la educación como práctica social transformadora está comprometida con la construcción de una sociedad más justa e igualitaria y con la formulación de alternativas, y esto requiere que la práctica educativa supere el marco escolar y tenga en cuenta los espacios educativos no formales y ámbito informales como los medios de comunicación. Y que el ejercicio de la ciudadanía se oriente a lograr una "democracia real" capaz de dar respuesta a las necesidades de las personas y que se articule a partir de movimientos que propugnan una radical reinvención de la democracia a nivel global y local, con nuevas instituciones internacionales que regulen las cuestiones mundiales, así como una profundización de la democracia electoral y la expansión de fórmulas participativas en el plano nacional, regional o local.

Y esto sólo pueden hacerlo en estrecha relación con las ONG, con los movimientos sociales y con las organizaciones de la sociedad civil que integran redes internacionales y que desarrollan grandes campañas de incidencia política y de resistencia global, que intentan ir de la "Protesta a la propuesta". Organizaciones que plantean propuestas realizables a corto y medio plazo, desde un enfoque más integral, a partir de la vinculación creciente entre investigación, movilización social, acción sociopolítica y educación para la ciudadanía global.

Se trata de una ciudadanía cada vez más consciente de la necesidad de la gestión colectiva de los problemas comunes de carácter global, que adoptan una conciencia planetaria, en 
las que las cuestiones globales se han convertido en parte de las experiencias locales del día a día. En definitiva, se trata de estar comprometido con lo local y lo global al mismo tiempo.

\section{Conclusiones}

En este artículo se ha hecho una reflexión sobre el papel de la educación en el actual contexto internacional, marcado por profundos cambios y por un proceso de involución. Se han presentado diversos marcos interpretativos que explican la realidad y que enfrentan a la educación ante el dilema de ser un factor de reproducción de los valores dominantes que legitiman el statu quo, o bien convertirse en un motor de cambio que busca transformar la realidad injusta y desigual. La educación para la ciudadanía global se presenta como una oportunidad en el marco de la Agenda 2030 para promover la solidaridad, la justicia y la igualdad. Es una propuesta totalmente disruptiva que ofrece alternativas y que es el resultado de un consenso internacional en torno al desarrollo económico, social y ambiental y la afirmación de la paz y seguridad como un pilar esencial del desarrollo.

La educación para la ciudadanía global se asienta sobre una concepción integral de la educación, que implica a múltiples actores y que se lleva a cabo su acción en el ámbito local e internacional. La idea de lograr un futuro más justo, equitativo y sostenible se incorpora a la educación y es parte de esta agenda. La necesidad de" localizar" la Agenda en relación a las capacidades y retos de cada país y cada localidad y territorio abre extraordinarias oportunidades para dar paso a agendas a la vez locales y globales en el ámbito educativo. Y es en este marco, en el que la conformación de una ciudadanía global, construida desde abajo, desde las iniciativas de las organizaciones de la sociedad civil y desde la comunidad educativa, juegan un papel fundamental para afrontar estos problemas globales, que forman parte de las experiencias locales del día a día.

\section{Referencias}

Berger, P. y Luckman, T. (1968). La construcción social de la realidad. Buenos Aires: Amorrortu.

Delors, J. (1997). La educación encierra un tesoro. Informe a la UNESCO de la comisión internacional sobre la educación para el siglo XXI. París. UNESCO.

De Paz, D. (2007). Escuelas y educación para la ciudadanía global. Una mirada transformadora. Barcelona: Oxfam.

Fernández de Castro, P. y González-Paramo, A. (Dirs.). (2019). La franquicia antimigracion. Como se expande el populismo xenófobo en Europa. Madrid: Fundación porCausa.

Gimeno, J. (2005). La educación que aún es posible. Barcelona: Paidós.

Lederach, J. P. (2007). La imaginación moral. El arte y el alma de la construcción de la paz. Bilbao: Bakeaz y Gernika Gogoratuz.

Magallón, R. (2019). Un faking newes. Como combatir la desinformación. Madrid: Piramide.

Mesa, M. (2018). El ODS 16 sobre paz, seguridad y gobernanza: Desafíos conceptuales, seguimiento y evaluación. En M. Mesa (Coord.), Derechos humanos y seguridad internacional: Amenazas e involución. Anuario de CEIPAZ $2017-2018$ (pp. 37-52). Madrid: CEIPAZFundación Cultura de Paz. 
Maquieira, V. (2001). Género, diferencia y desigualdad. En E. Beltrán y V. Maquieira, (Eds), Feminismos: Debates teórico Contemporáneos (pp. 87-99). Madrid: Alianza.

Morin, E. (1999). Los siete saberes necesarios para la educación del futuro. París: UNESCO.

Novo, M. (2017). El papel de arte y de la educación. Cambiar en tiempos de incertidumbre. En S.M.A.R.T. (Coord.), Caminos hacia la sostenibilidad (pp. 256-261). Madrid: Acciona.

Prats, F. (2017). La gran encrucijada. Sobre la crisis ecosocial y el cambio de ciclo histórico. Madrid: Libros en Acción.

Stanley, J. (2019). Our increasingly fascist public discourse. Recuperado de https://www.projectsyndicate.org/onpoint/our-increasingly-fascist-public-discourse-by-jason-stanley-2019$01 /$ spanish?barrier=accesspaylog

Sampedro, V. (2018) Dietética digital. Para adelgazar al gran hermano. Barcelona: Icaria.

Sanahuja, J. A. (2018). Crisis de globalización, crisis de hegemonía: Un escenario de cambio estructural para América Latina. En A. Serbin (Coord.), América Latina y el Caribe frente a un nuevo orden mundial: Poder, globalización y respuestas regionales (pp. 37-68). Barcelona: Icaria editorial.

Santos, B. de S. (2011). Epistemologías del sur. Utopía y Praxis Latinoamericana, 16(54), 17-39.

Vallespin, F. y Bascuñán, M. M. (2017). Populismos. Madrid: Alianza.

\section{Breve CV de la autora}

\section{Manuela Mesa}

Doctora en Sociología y Antropología por la Universidad Complutense de Madrid. Pedagoga especializada en educación para la paz y el desarrollo y en estudios de paz. Es directora del Centro de Educación e Investigación para la Paz (CEIPAZ), de la Fundación Cultura de Paz, presidida por Federico Mayor Zaragoza Y Codirectora del Instituto Universitario DEMOSPAZ-UAM. De 2007 a 2013 fue Presidenta de la Asociación Española de Investigación para la Paz (AIPAZ). Vicepresidenta y cofundadora de WILPF-España desde 2011 hasta la actualidad. Especialista en Investigación para la Paz (Peace Research), en educación para la paz, y en educación para el desarrollo. Ha dirigido y llevado a cabo diversos proyectos de investigación sobre violencia transnacional y prevención de conflictos en América Latina, en los casos de Colombia y América Central. También ha realizado diversos estudios sobre educación para el desarrollo en el ámbito español y europeo. ORCID ID: https://orcid.org/0000-0003-0495-6117. Email: mmesa@ceipaz.org 